\title{
Expression and Simple Purification Strategy for the Generation of Anti- microbial Active Enterocin P from Enterococcus faecium Expressed in Escherichia coli ER2566
}

\author{
Thu Ngoc Le 1, Thi Huyen Do 1, Thanh Nhan Nguyen 1, Ngoc Tan Tran 1, Sven Olof Enfors ${ }^{2}$, Nam Hai \\ Truong 1,*
}

${ }^{1}$ Institute of Biotechnology, Vietnam Academy of Science and Technology, 18- Hoang Quoc Viet, Cau Giay Ha Noi, VIETNAM ${ }^{2}$ KTH-Biotechnology, Royal Institute of Technology, Rolagstullsbacken, S-10691, Stockholm, SWEDEN

* Corresponding author: Nam Hai Truong, Institute of Biotechnology, Vietnam Academy of Science and Technology, Ha Noi, VIETNAM. Tel: +840437917980, Fax: +84-0437560339, Email: tnhai@ibt.ac.vn

Received: July 25,2014; Revised: September 18,2014; Accepted: November 012014

\begin{abstract}
Background: Enterocin-P of Enterococcus faecium P13 (EntP) is of great interest as a food preservative and medicine due to its non-toxicity and broad antimicrobial spectrum at various $\mathrm{pH}$, as well as its excellent thermal stability. However, recombinant production of EntP is still in laboratory because of low productivity and complex purification process.

Objectives: In this study, we aimed to develop efficient methods for high-level expression and convenient purification of the recombinant EntP.

Materials and Methods: An artificially synthesized gene (entP) of 132 bp encoding mature enterocin P of E. faecium P13 was cloned in plasmid pTWIN1 under the control of an inducible T7lac promoter for expression of fusion protein EntPMxe GyrA mini-intein-chitin binding domain (CBD) (abbreviated by EntP-Int-CBD) in E. coli. Recombinant EntP was released from the fusion protein by DTT digestion and cleaned by distilled water and checked for anti-bacterial activity. Results: The fusion protein was highly expressed in insoluble form in $E$. coli at $37^{\circ} \mathrm{C}$ with $0.05 \mathrm{mM}$ IPTG induction. The insoluble fusion protein EntP-Int-CBD was easily prepared by cell sonication and centrifugation to remove soluble contaminants. The repeat washing steps with Triton X-100 were applied to reduce contaminants. After DTT-induced selfdigestion in urea $4 \mathrm{M}$, the EntP released from the fusion protein was insoluble in water and easier to be separated from soluble Int-CBD by centrifugation. The recombinant peptide was soluble in $20 \% 2$-propanol in $0.1 \%$ trifluoroacetic acid (TFA) and exhibited strong anti- Listeria monocytogenes and Staphylococcus aureus activities.

Conclusions: This study is the first report providing a simple, quick and straight forward procedure for heterologous production of functional and pure Enterocin P without using any chromatography columns in the purification process.

Keywords: Antimicrobial activity; E. coli expression; Enterocin P; Mxe GyrA mini-intein-chitin binding domain (CBD); Purification
\end{abstract}

\section{Background}

In recent years, the use of bacteriocins in food biopreservation has gained growing interest, because it holds promise for reducing the need for chemical preservatives and high-intensity heat treatments, thereby potentially resulting in foods more "naturally" preserved and richer in nutrients.

Similar to other bacteriocins, the pediocin-like bacteriocin, termed enterocin-P (EntP), produced by $E$. faecium P13 (originally isolated from a Spanish dry fermented sausage), is considered as a safe food biopreservative because it is quickly degraded by proteases in the gastrointestinal tract (1). Compared to most common other subclass IIa bacteriocins, EntP exhibits a broad spectrum of antimicrobial activity against a broad spectrum of spoilage bacteria such as Ent. faecalis, Staphylococcus carnosus, Clostridium sporogenes, C. tyrobutyricum, and Propionibacterium spp. and food-borne pathogens such as Listeria monocytogenes, Clostridium perfringens, C. botulinum, and $S$. aureus (3). EntP causes antimicrobial activity by forming specific potassium ion-conducting pores in the cytoplasmic membranes of target cells, which in turn causes a rapid and drastic efflux of the intracellularly accumulated potassium ions thus impairing the electrochemical transmembrane potential $(4,5)$. 
The EntP is very stable. It tolerates (i) heating at $100^{\circ} \mathrm{C}$ for $60 \mathrm{~min}$ or $121^{\circ} \mathrm{C}$ for $15 \mathrm{~min}$; (ii) exposure to $\mathrm{pH}$ between 2 and 11 for $24 \mathrm{~h}$ at $25^{\circ} \mathrm{C}$; (iii) repeated freeze-thawing cycles as well as lyophilization; and (iv) long-term storage at $4^{\circ} \mathrm{C}$ and $-20^{\circ} \mathrm{C}$ without losing the antimicrobial activity (3). Accordingly, EntP holds great promise as a natural preservative in the food industry, but also in medicine as an antibiotic supplement $(3,6,7)$. However, since E. faecium may carry antibiotic-resistant genes and/or genes encoding potential virulence factors and is therefore not the ideal production host. Hence, many efforts on heterologous production of functional EntP has instead been concentrated to hosts other than E. faecium (8-11).

The enterocin-P structural gene (ent $P$ ) encodes a 71 amino acid (aa) prepeptide $(7.5 \mathrm{kDa})$ containing a 27 aa long signal peptide that is removed proteolytically upon secretion, yielding the 44 aa mature bacteriocin (approximately $4.6 \mathrm{kDa})(4,11)$. Among expression systems investigated for bacteriocin production, $E$. coli has been reported to yield around $1 \mathrm{~g} /$ lit culture (2), however up to now there has been no truly successful attempt in the case of EntP.

The direct expression of small peptides such as enterocin-P in E. coli is often inefficient as such peptides frequently are subject to in vivo degradation by host cell proteases, which significantly reduces the yield. To alleviate this, short peptides can be expressed in fusion to enhance the stability and then released by proteolytic or chemical reagents $(11,12)$. Previously, EntP has been expressed in E. coli in fusion with thioredoxin (Trx), or together with the EntP immunity gene or in a prepeptide format (10) with limited success. Trx-(His) ${ }_{6}$-EntRP fusion protein was purified on an immobilized metal ion affinity column and subsequently cleaved with enterokinase to release only a few functional EntP (10), while in the two other cases the proteins were secreted into the medium at very low concentrations, and then recovered by immunoaffinity chromatography or a laborious procedure involving ammonium sulfate precipitation, desalting and three consecutive chromatography steps (cation exchange, hydrophobic interaction chromatography and reversephase chromatography) (9).

Inteins are self-splicing proteins that have been used in recombinant protein expression and purification procedures and are readily available through commercial vectors such as pTWIN1, pTWIN2 etc. The inteins play a role in formation of a well- designed construct that is stably expressed and are non-toxic to host cells $(7,13,14)$. Ssp intein cleavage is initiated by low $\mathrm{pH}$, and Mxe GyrA intein cleavage by 1,4-dithiothreitol (DTT). The Mxe GyrA intein has been used successfully to express small peptides in fusion proteins that form granules or inclusion bodies. These fusion proteins are easily purified by collecting the insoluble fraction, and following solubilization, then effectively digested by addition of DTT to release the functional peptides (15-17). This novel technique is considered as a powerful and competitive alternative to affinity chromatography and conventionally used in protein purification.

\section{Objective}

In this study, Mxe GyrA intein linked with a chitin binding domain (CBD) tag was used as a fusion partner for the production of an insoluble fusion protein EntP-Int-CBD in E. coli. For the first time, we introduced a simple procedure (without using any chromatography) for obtaining recombinant EnP with antibacteria activity.

\section{Materials and Methods}

\subsection{Strains and Reagents}

E. coli $\mathrm{DH} 5 \alpha$ [end A1 rec A1 hsd R17 sup E44 gyp A96 thi-1 relA1s lac U169 ( $\$ 80$ lacZM15)] (Invitrogen) and E. coli ER2566 [fhuA2 [lon] ompT gal sulA11 R(mcr-73::miniTn10-TetS)2 [dcm] R (zgb210::Tn10-TetS) endA1 $\Delta$ (mcrC-mrr) 114::IS10] (New England Biolabs, USA) were utilized for cloning and expression of the entP gene. L. monocytogenes provided by Institute of Post Harvest Biotechnology, Vietnam and $S$. aureus provided by Chemistry Institute, Vietnam Academy of Science and Technology, Vietnam were used as indicators to test the antimicrobial activity of recombinant EntP. pJET1/blunt cloning vector was purchased from Fermentas, and expression plasmid pTWIN1 was obtained from New England Biolab (NEB). Restriction endonucleases and ligase used in the cloning procedures (Fermentas, USA), and anti-chitin binding domain serum (NEB) for detection of the fusion proteins, were all utilized according to the manufacturer's recommendations. The oligonucleotide primers were synthesized by Integrated DNA Technologies, Inc, USA.

\subsection{Construction of Plasmid pTWINentP}

Based on the sequence of $E$. faecium $\mathrm{P} 13$ entP gene (GenBank: AF005726.1), two pairs of primers were designed. One overlapping forward primer, 5' 
tcttctcgaggacccggctacTcgttcatatggtaatggtgtttattgtaataa tagtaaatgTtgggttaactggggagaagctaaagag- $3^{\prime}$, and the other overlapping reverse primer, $5^{\prime}$-aattgggccgcttaatgtcccatacctgc caaaccagaagcccagccactaataacgattcctgcaatattctctttagcttctcccca-3' (overlapping nucleotides are underlined). Seventeen additional nucleotides at the 5' terminal end of the forward primer were included to allow for expression of secreted EntP in yeast $P$. pastoris. Two nucleotides in the forward primer (22T, 64T; in capital letters) result in two silent nucleotide substitutions in the entP gene coding for mature EntP, and were included to avoid rare codons. The second set of EntP specific primers was entP forward: $5^{\prime}$-gcatatggctactcgttcttatggtaa-3' (NdeI underlined) and entP reverse: 5' -tgctcttctgcaatgtcccatacctgccaa-3' (SapI underlined).

The first two overlapping primers were used to synthesize entP based on the gene sequence from $E$. faecium P13, using Klenow polymerase (large fragment of DNA polymerase I) in a primer extension reaction using the following program: denaturation $\left(94^{\circ} \mathrm{C}\right.$ for 2 $\mathrm{min})$, annealing $\left(55^{\circ} \mathrm{C}\right.$ for $\left.2 \mathrm{~min}\right)$ and then Klenow was added for elongation $\left(37^{\circ} \mathrm{C}\right.$ for $\left.30 \mathrm{~min}\right)$, ending with an extension at $37^{\circ} \mathrm{C}$ for $7 \mathrm{~min}$ and finally keeping the product at $4^{\circ} \mathrm{C}$. The obtained product appeared as a smear of diverse DNA fragments lengths (Figure 1A). Using Taq DNA polymerase, entP was then PCR amplified from this with primers entP forward and entP reverse $\left(94^{\circ} \mathrm{C}\right.$ for $2 \mathrm{~min} ; 25$ cycles of $94^{\circ} \mathrm{C}$ for 1 $\min , 62^{\circ} \mathrm{C}$ for $1 \mathrm{~min}$, and $72^{\circ} \mathrm{C}$ for $30 \mathrm{~s}$, with a final hold for an extra $7 \mathrm{~min}$ at $72^{\circ} \mathrm{C}$ ).

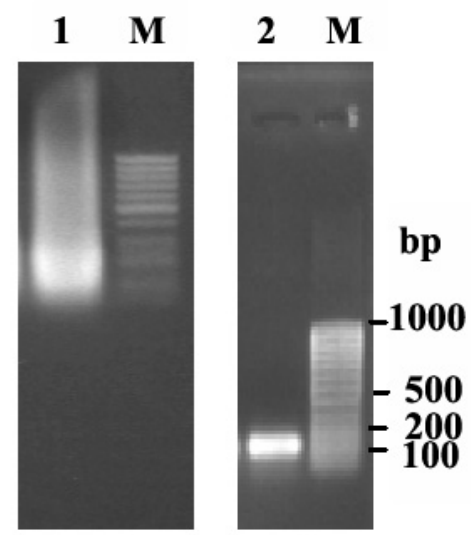

Figure 1. Analysis of in vitro synthesized entP gene fragments on $2 \%$ agarose gel. 1: entP synthesized by primer extension of overlapping entP-encoding oligonucleotides; 2: entP amplicon following PCR with entP-specific primers and the entP-fragment from lane 1 as template. M: standard 50 bp DNA ladder (Fermantas, USA)
The obtained PCR product was cloned into pJET1/blunt, sequenced, digested with NdeI and SapI, and finally ligated into NdeI/SapI-linearized pTWIN1, yielding pTWINentP. This expression vector was transformed into E. coli ER2566, and the transformants were selected on LB agar plates containing 100 $\mu \mathrm{g} . \mathrm{ml}^{-1}$ ampicillin.

\subsection{Expression of Recombinant EntP-Int-CBD in E. coli ER2566}

A single-colony transformant was inoculated into $10 \mathrm{ml} \mathrm{LB}$ (supplemented with $100 \mu \mathrm{g} \cdot \mathrm{ml}^{-1}$ ampicillin; LBA), and grown overnight at $37^{\circ} \mathrm{C}$ in a rotary shaker (200 rpm). One percent of the overnight culture was then transferred to $500 \mathrm{ml}$ of fresh LBA in two $2 \mathrm{~L}$ flasks and cultivated at $37^{\circ} \mathrm{C}$. When the optical density (OD600) reached 0.6-0.8, IPTG was added to a final concentration of $0.05 \mathrm{mM}$ to induce expression of the fusion protein EntP-Int-CBD and the cultivation was continued for $3 \mathrm{~h}$ at $37^{\circ} \mathrm{C}$. The cells were harvested by centrifugation at $6000 \mathrm{rpm}$ for $10 \mathrm{~min}$ at $4^{\circ} \mathrm{C}$, and the cell pellets were stored at $-80^{\circ} \mathrm{C}$ until use. Various IPTG concentrations $(0,0.001,0.01,0.05,0.1,0.5,1$, 5 , and $10 \mathrm{mM}$ ) were also tested to identify the optimal IPTG concentration to be used for the EntP-Int-CBD production. The expression products were analyzed by SDS-PAGE and Western blot.

\subsection{Gel Electrophoresis and Western Blots}

Tricine-SDS-PAGE for protein analysis was followed by a description of Schägger and Von Jagow (1987) (18). Gel was then equilibrated with transblot buffer (2.93 g.1-1 glycine, 5.81 g.1.$^{-1}$ Tris-HCl, 0.37 g.11SDS, $20 \% \mathrm{v} / \mathrm{w}$ methanol) for $30 \mathrm{~min}$ followed by blotting to equilibrated PVDF membranes (Westran ${ }^{\circledR}$ $\mathrm{S}$, Whatman) for $75 \mathrm{~min}$ at $25 \mathrm{~V}$ (Trans-blot ${ }^{\circledR} \mathrm{SD}$ Semidry transfer cell machine, Bio-Rad). The membranes were blocked overnight in TBS (61 g.1- Trizma base, 146.1 g. $\left.1^{-1} \mathrm{NaCl}, \mathrm{pH} 7.4\right)$ containing $5 \% \mathrm{w} / \mathrm{v}$ skim milk powder and then washed for $5 \mathrm{~min}$ in TTB. The washed membrane was then incubated for $3 \mathrm{~h}$ in TBS containing $5 \% \mathrm{w} / \mathrm{v}$ skim milk powder and 2 $\mu 1 . \mathrm{ml}^{-1}$ Anti-CBD monoclonal antibody (BioLabs, USA). The membrane was then washed three times for 5-10 min in TTBS afterward incubated for $3 \mathrm{~h}$ in TBS containing $5 \% \mathrm{w} / \mathrm{v}$ skim milk powder and $1 \mu 1 . \mathrm{ml}^{-1}$ HRPO conjugated goat anti-mouse antibody (Sigma, USA). Detection was achieved by the addition of freshly prepared solution (25 ml TBS $15 \mathrm{ml} \mathrm{H}_{2} \mathrm{O}_{2}$ mixed with $15 \mathrm{mg}$ 4-chloro-1-naphtol in $5 \mathrm{ml}$ methanol). 


\subsection{Purification of EntP from EntP-Int-CBD}

The cell pellets obtained as above were thawed at room temperature and then resuspended in $30 \mathrm{ml}$ of lysis buffer B1 (20 mM Tris-HCl, pH 8, 1 mM EDTA, $500 \mathrm{mM} \mathrm{NaCl}$ ) supplemented with $0.1 \mathrm{mg} \cdot \mathrm{ml}^{-1}$ lysozyme and $0.5 \mathrm{mM}$ PMSF. The cell mixture was incubated at $37^{\circ} \mathrm{C}$ for $1 \mathrm{~h}$ before the cells were disrupted by sonication on ice bath (10 pulses, $30 \mathrm{~s}$ each at $100 \mathrm{~W}$ with $20 \mathrm{~s}$ intermission). Fusion protein EntPInt-CBD, in the form of inclusion bodies, was recovered by centrifugation at $12000 \mathrm{rpm}$ for $30 \mathrm{~min}$. The inclusion bodies were washed, first with buffer B1, then with buffer B1 supplemented with $2 \mathrm{M}$ urea and $10 \mathrm{mM}$ DTT, and finally with buffer B1supplemented with $0.1 \%$ triton $\mathrm{X}-100$. These washing steps helped to remove some contaminants, and the washed inclusion bodies were subsequently solubilized in $30 \mathrm{ml}$ of denaturing/solubilizing buffer (20 mM Tris- $\mathrm{HCl}, \mathrm{pH} 8,1$ mM EDTA, $500 \mathrm{mM} \mathrm{NaCl}$, urea $8 \mathrm{M}$ ).

After denaturation, the solubilized inclusion bodies were diluted to $4 \mathrm{M}$ urea with buffer B1, and selfcleavage of the fusion protein EntP-Int-CBD was induced by addition of $50 \mathrm{mM}$ (final concentration) DTT (Mxe Intein cleavage is initiated by DTT). The mixtures were incubated at room temperature for $16 \mathrm{~h}$ and samples withdrawn for subsequent analysis on $16 \%$ tricine SDS-PAGE. The DTT-cleaved mixture was then precipitated with ammonium sulfate $(75 \%$ saturation) and kept at $4^{\circ} \mathrm{C}$ with stirring for $2 \mathrm{~h}$. After centrifugation at $12000 \mathrm{rpm}$ at $4^{\circ} \mathrm{C}$ for $20 \mathrm{~min}$, the pellet was washed extensively with deionized water to remove most contaminants (EntP remained insoluble in water), and finally dissolved in buffer containing $20 \%$ 2-propanol in aqueous $0.1 \%$ (vol/vol) trifluoroacetic acid (TFA), thus yielding highly pure and soluble EntP.

\subsection{Direct antimicrobial activity assay of recombinant EntP on Tricine SDS-PAGE}

The DTT-cleaved mixture was subjected to $16 \%$ Tricine-SDS-PAGE as described by Schägger \& von Jagow (1987) (18) followed by a slightly modified direct antimicrobial activity assay (19) using $S$. aureus as the indicator strain. More specifically, after electrophoresis, the gel was stained briefly ( $15 \mathrm{~min}$ ) with Coomassie blue and then destained until the background stain disappeared. The gel was then equilibrated in deionized water for about $3 \mathrm{~h}$ and carefully loaded on a LB agar plate. After that, $5 \mathrm{ml}$ of tempered LB soft agar $(0.6 \%$ agar $)$ containing $10^{4}$ cells of indicator microorganism $(S$. aureus) was poured over the gel, and the plate was incu- bated at $4^{\circ} \mathrm{C}$ for $2 \mathrm{~h}$ prior to overnight incubation at $37^{\circ} \mathrm{C}$ before the inhibition zone was examined.

\subsection{Detection of antimicrobial activity of partially} purified recombinant EntP by an agar well diffusion test (ADT)

The test of the antimicrobial activity of partially purified recombinant EntP, an agar well diffusion test, described by Toba et al. (1991) (20), using S. aureus as the indicator microorganism was used. In brief, a solidified LB agar plate was overlaid with $5 \mathrm{ml} \mathrm{LB}$ soft agar seeded with $100 \mu 1$ indicator organisms (approximately $10^{7}$ cells). Wells (5 $\mathrm{mm}$ diameter) were punched into this plate, and $50 \mu 1$ of two-fold serial dilutions of the purified EntP was added to each well. The plate was then incubated at $4^{\circ} \mathrm{C}$ for $2 \mathrm{~h}$ prior to overnight incubation at $37^{\circ} \mathrm{C}$ before the presence of potential inhibition zones around the wells was checked. The bacteriocin activity was expressed as AU $\mathrm{ml}^{-1}$ (arbitrary unit per milliliter) defined as the reciprocal of the highest two-fold dilution showing inhibitory action against the indicator strain.

\section{Results}

\subsection{Construction and Expression of Recombinant} EntP-Int-CBD in E. coli ER2566

By conducting a primer extension reaction on a pair of overlapping oligonucleotides encoding EntP, using klenow polymerase (without 5 '-exonuclease activity), EntP fragments in various sizes were generated. However, a more concentrated band that appeared to be of the correct gene entP size (132 bps) was also visible (Figure 1A). In a subsequent step, this fragment was PCR amplified by specific primers (Figure 1B) and eventually cloned into pTWIN1, yielding the expression vector pTWINentP, which expresses the chimeric fusion protein EntP-Int-CBD under the control of a T7lac promoter. Upon IPTG induction, E. coli ER2566 harboring pTWINentP generated EntP-IntCBD with an approximate size of $33.5 \mathrm{kDa}$ (Figure 2A). The EntP-Int-CBD fusion accounted for nearly $70 \%$ of the total cell proteins, according to the analysis in VersaDoc Imaging system Model 4000 (BioRad, USA) by Quantity One, Version 4.6.1 (Bio-Rad, USA).

However, over-expression of the fusion protein mainly resulted in the formation of biologically inactive aggregates (Figure 2C, Figure 3 ). When using low temperature $\left(15^{\circ} \mathrm{C}\right)$ cultivation, over $50 \%$ of EntP-IntCBD still remained as inclusion bodies (data not 
$\mathbf{A}$

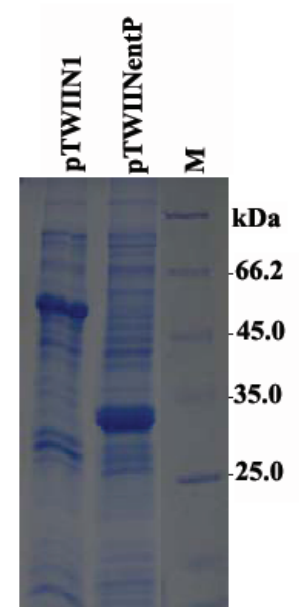

B

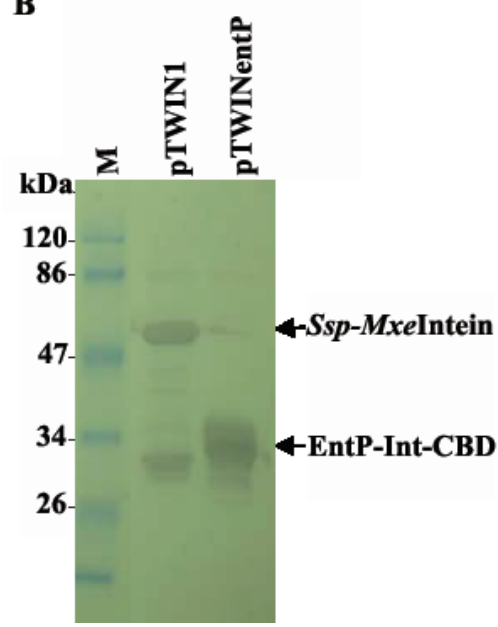

C

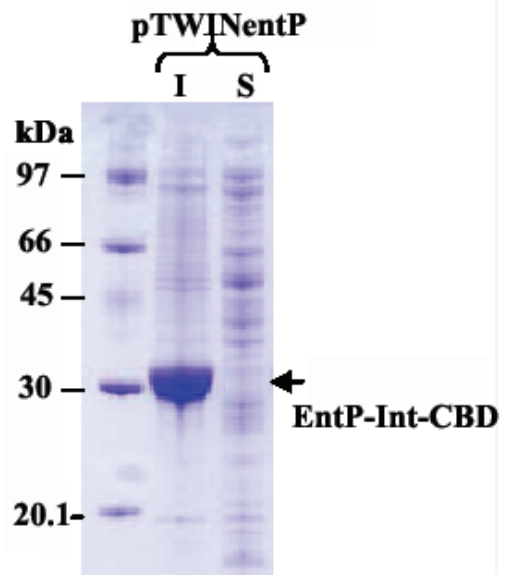

Figure 2. A: SDS-PAGE analysis of proteins expressed in recombinant E. coli ER2566 cells harboring pTWIN1, pTWINentP B: Western blot analysis of recombinant protein by anti-CBD monoclonal antibody (BioLabs) and C: SDSPAGE analysis of soluble, insoluble protein fractions expressed in recombinant $E$. coli ER2566 cells harboring pTWINentP. pTWIN1, pTWINentP: Proteins from E. coli ER2566 harboring plasmid pTWIN1, pTWINentP respectively; M: standard proteins (Fermentas, USA). Spp-Mxe Intein is fusion Spp intein and Mxe intein was expressed in E. coli ER2566 harboring pTWIN1

shown). High IPTG concentration (up to $10 \mathrm{mM}$ ) did not affect on the growth of the recombinant strain, however, $0.05 \mathrm{mM}$ IPTG is the most suitable inducer concentration for expression of the recombinant EntPInt-CBD from pTWIN1-entP in E. coli ER2566 (data not shown). The expressed chimeric proteins were also confirmed by Western blot using anti-CBD serum (Figure 2B).

The released EntP from DTT digested EntP-IntCBD fusion seemed to conform well with the molecular weight $(4.6 \mathrm{kDa})$ of mature EntP from E. faecium
P13 as revealed by the Tricine SDS-PAGE analysis (Figure 3A). An initial experiment showed that the released recombinant EntP also exhibited strong activity against $S$. aureus as demonstrated by the clear growth inhibition zone around the band of EntP (Figure 3B) in polyacrylamide gel. This result suggests that recombinant EntP indeed could be obtained in a biologically active form after cleavage with DTT.

\subsection{Purification of biologically active EntP from EntP-Int-CBD}

$\mathbf{A}$

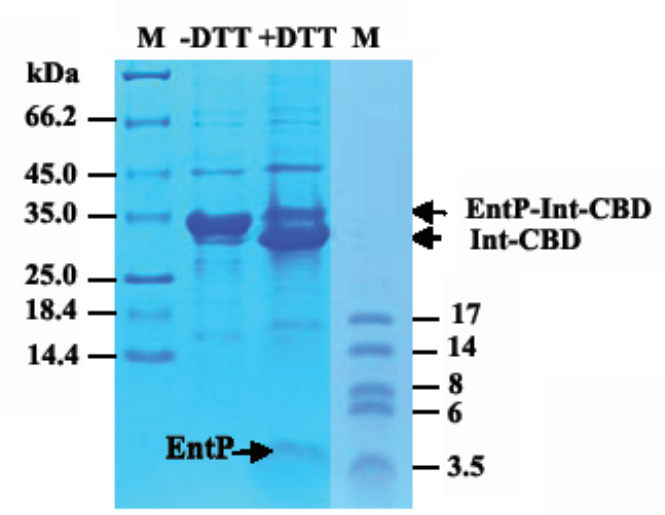

B

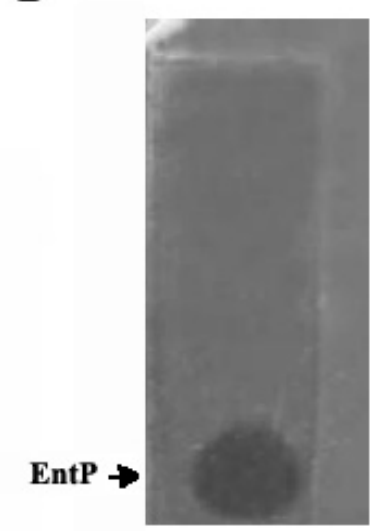

Figure 3. A: 16\% Tricine SDS-PAGE analysis of DTT digested EntP-Int-CBD in the presence of $4 \mathrm{M}$ urea, B: Direct in gel (Tricine SDS-PAGE), antimicrobial activity assay of the recombinant EntP released from the fusion protein, using $S$. aureus as the indicator strain. M: MW standard proteins (Fermentas) 
In this study, buffer B1 (20 mM Tris-HCl, $\mathrm{pH} 8,1$ mM EDTA, $500 \mathrm{mM} \mathrm{NaCl}$ ) supplied with lysozyme was used in combination with sonication to release the intracellular protein content of the recombinant $E$. coli cells. The inclusion bodies of EntP-Int-CBD obtained from this step were relatively pure (over than $85 \%$ purity as determined by Quantity One software). Nevertheless, this chimeric protein was still rinsed in three consecutive steps by buffer containing $2 \mathrm{M}$ urea and $10 \mathrm{mM}$ DTT, then by buffer supplemented with $0.1 \%$ Triton X-100 (Figure 4A).

After digestion with DTT, and subsequent precipitation with ammonium sulfate, Int-CBD now became soluble in water and visible in the SDS-PAGE analysis (Figure 4B). However, as EntP remained insoluble in water, several washings in water were required for efficient removal of Int-CBD and other impurities from the insoluble EntP (Figure 4B).

The final step in the purification scheme was to find an appropriate solvent composition that would enable recovery of EntP in a soluble and active form. EntP is a peptide with strong cationic and hydrophobic characteristics/properties and is prone to form aggregates (21). However, acidic solvents have recently been reported to be useful for improving the solubility of EntP, thereby enabling its purification (9). Accordingly, in this study the recovery and isolation of functional EntP was investigated by trying to dissolve the precipitate in three different acidic buffers, i.e., 20
mM sodium phosphate buffer, $\mathrm{pH}$ 5.8; 85\% 2-propanol $\mathrm{v} / \mathrm{v}$ in water, $\mathrm{pH} 2.0$; and 20\% 2-propanol in aqueous $0.1 \%$ TFA. As is visible in Figure 4C, EntP was easily solubilized in $20 \%$ 2-propanol in aqueous $0.1 \%$ TFA. However, some of the impurities went into solution, as indicated by the faint bands in the SDS-PAGE analysis (Figure 4C). The other two buffer compositions that were tested failed to solubilize EntP (Figure 4C).

\subsection{Purified EntP exhibits antimicrobial activity}

$L$. monocytogenes and $S$. aureus were used as indicator strains in the antimicrobial activity assay. In these experiments, different serial dilutions of purified and soluble EntP were loaded in wells, punched into agar plates seeded with indicator bacteria. The plates were first incubated at $4^{\circ} \mathrm{C}$ for 2 hours to allow EntP to diffuse well into the agar before the plates were incubated overnight at $37^{\circ} \mathrm{C}$ to promote cell growth, and finally examined for potential cell growth inhibition. The EntP clearly inhibited the growth of both strains but to different degrees (Figure 5); being most potent against $L$. monocytogenes. When increasing the duration of the initial diffusion step to overnight at $4^{\circ} \mathrm{C}$, the activity of EntP increased nearly 1000-fold (data not shown). In addition, mixtures of EntP and the pathogenic bacteria that were kept at $4^{\circ} \mathrm{C}$ for different durations, before they were spread on ordinary LB plates, showed that the cell viability dropped dramatically for incubation times exceeding three hours. This indicated
$\mathbf{A}$

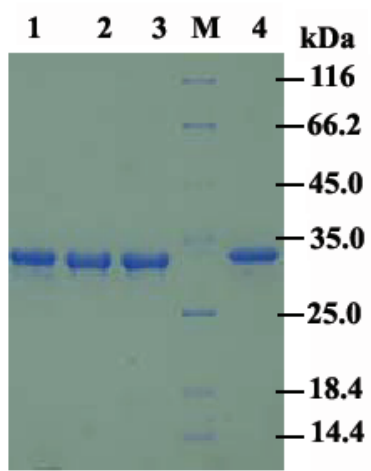

B

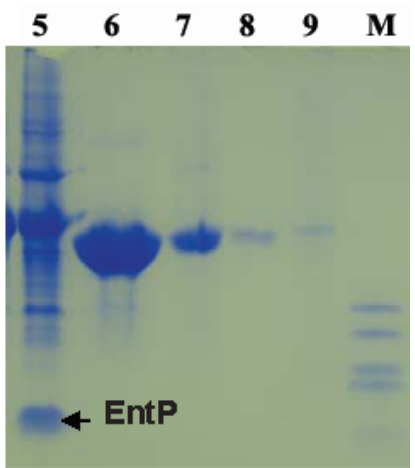

C

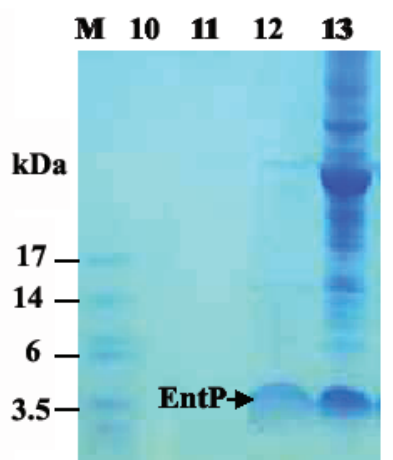

Figure 4. A: SDS-PAGE analysis of protein fractions from insoluble EntP-Int-CDB washing steps, B: The soluble Int-CBD removing and C: Solubilizing process of EntP. 1: The insoluble EntP-Int-CBD from whole-cell lysates; 2-4: The insoluble EntP-Int-CBD was washed by buffer B1, B1 supplemented with $2 \mathrm{M}$ urea and $10 \mathrm{mM}$ DTT, and B1 supplemented with $0.1 \%$ Triton X-100 respectively; 5, 13: the DTT digested EntP-Int-CBD was precipitated by ammonium sulphate; 6-9: the soluble Int-CBD separated from EntP through four consecutive water washes of DTT digested EntP-Int-CBD respectively. 1012: The EntP obtained after treatment with $20 \mathrm{mM}$ sodium phosphate buffer $\mathrm{pH}$ 5.8; 85\% 2-propanol pH 2.0 in water (v/v), or 20\% 2-propanol in aqueous $0.1 \%$ TFA respectively 

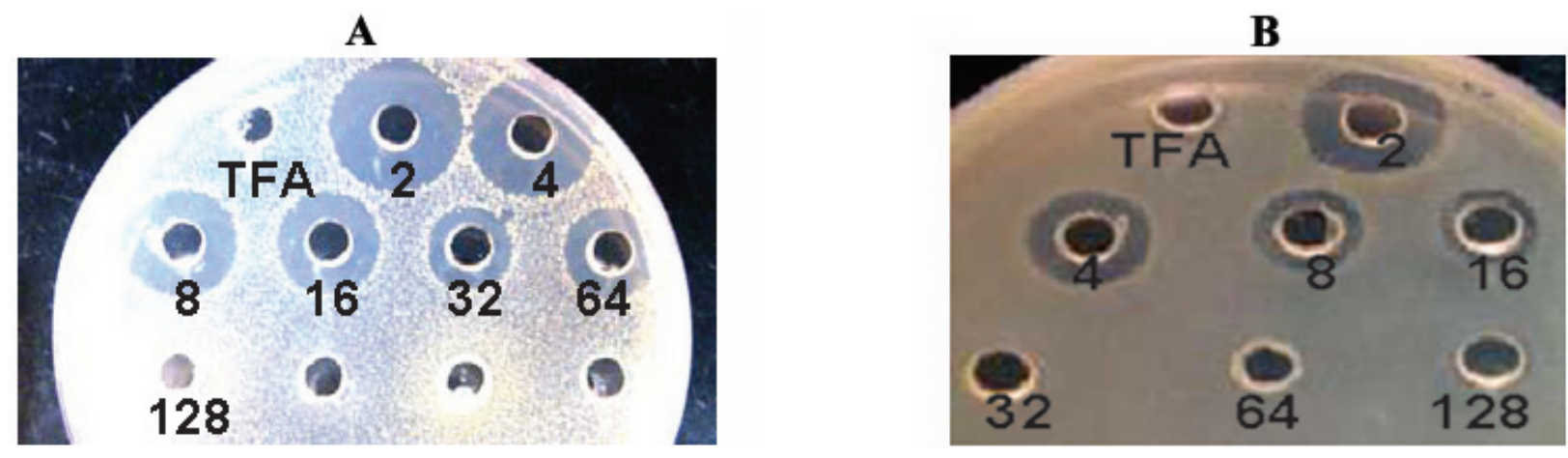

Figure 5. A: Agar well diffusion test to detect antimicrobial activity of purified recombinant EntP against L. monocytogenes, and B: S. aureus. The numbers indicate the dilution values; TFA: $20 \%$-propanol in aqueous $0.1 \%$ TFA, used as a negative control

that EntP not only has the ability to inhibit bacterial cell growth but also to kill the bacteria (data not shown).

\section{Discussion}

The fusion protein EntP-Int-CBD was expressed at high levels in E. coli cells as inclusion bodies, and easily harvested from bacterial cell lysates by centrifugation and then solubilized in urea. Although the current approach resulted in an insoluble and inactive form of EntP fusion protein, this may actually be advantageous due to: (i) increased stability of the fusion protein by reducing the susceptibility to intracellular proteases; (ii) reduced toxicity caused by active EntP, thus the fusion proteins can be synthesized and accumulated at high concentration in the cells; (iii) avoiding premature cleavage in vivo since a correct fold conformation is required for the self-splicing activity of intein (22). For instance, in a previous study by Gutiérrez et al, the low level of EntP when expressed in its active form in E. coli was believed to indicate toxicity to the host cells (9).

Urea, DTT, Triton X-100 are the detergents that frequently are applied to reduce the levels of contaminants in insoluble proteins (or inclusion bodies). As mentioned earlier, target proteins fused with $M x e$ Intein and expressed from the pTWIN1 plasmid family can be cleaved at the N-terminus of intein by using thio-reagents such as DTT (15-17). We successfully digested the EntP-Int-CBD fusion protein with $4 \mathrm{mM}$ DTT to release functional recombinant EntP. Initially, we investigated different final urea concentrations (1, 2,4 , and $8 \mathrm{M}$ ) for cleavage of the fusion protein by DTT, and the best result was obtained in $4 \mathrm{M}$ urea (data not shown). However, a few percent of EntP-IntCBD was apparently not digested during the DTT- treatment. Perhaps, the efficiency could be improved by increasing the DTT concentration in the digestion reaction, however, this was not tested.

Upon the DTT-induced digestion, EntP was recovered as insoluble aggregates from the ammonium sulfate precipitated cleavage mixture, from which the remainder of the cleaved fusion protein/contaminants could be removed by washing in water. Finally, EntP was dissolved in buffer (20\% 2-propanol in aqueous $0.1 \%$ TFA) and exhibited strong antibacterial activity in various assays.

In a previous attempt by our research group, EntP was expressed as a C-terminal fusion to CBD-Ssp Intein from pTWIN1 (23). In agreement with the present study, CBD-Ssp Intein-EntP was also overexpressed in E. coli ER2566 and formed insoluble aggregates with molecular weight of ca. $35 \mathrm{kDa}$. However, subsequent attempts to release EntP through low $\mathrm{pH}$-mediated self-cleavage failed as the efficiency proved to be very low (23). Thus, active EntP was achieved in very limited amounts (data not shown). Moreover, we have also tried earlier alternative fusion partners, such as amylases of Saccharomyces fibuligera and Bacillus amyloliquefaciens, for expression of EntP in Pichia pastoris SMD1168 (24) and B. subtilis BS168 (25) using pPIC9- and pAC7-based vectors, respectively. A cleavage site for formic acid was engineered between EntP and the amylases. However, this strategy also failed, as we were unable to detect any target protein in an SDS-PAGE analysis (data not shown).

EntP has been expressed in different host strains, such as E. coli, Methylobacterium extorquens ATCC 55366, L. lactis, P. pastoris $(8,26)$. However, the strategies used for the production and/or purification in these cases will most likely only are of limited use 
in a large-scale production setting. This is because they (i) rely on the use of expensive enzymes for removal of the fusion tags, which is time consuming and costly; or (ii) result in low expression levels with several contaminants, which then requires several chromatography steps for capture and purification of the target protein, and therefore usually also poor yields. For instance, in one example, as little as $3.1 \%$ was recovered after purification starting from over $6 \mathrm{mg} / \mathrm{L}$ culture (9). In another example, EntP was produced in $P$. pastoris at relatively high levels (28.2 mg.L-1 culture), however, the recovery of functional EntP was complex, involving three steps including filtration, cationexchange and reverse-phase chromatography (11).

In conclusion, this study is the first reporting large amounts of over-expressed EntP as fusion proteins forming inclusion bodies, paired with a simple and efficient purification process that does not rely on the use of any chromatography steps to get anti-bacterially active, soluble recombinant EntP. We believe that the novel process for the expression and purification of highly active EntP, which we report here, will be of benefit in large-scale production of EntP as it may reduce the time and cost, compared to the previously described methods.

\section{Acknowledgements}

We would like to acknowledge Prof. Nguyen Thuy Chau (Institute of Agricultural Engineering and Post Harvest Technology, Vietnam) for the kind supply of L. monocytogenes, Dr. Pham Thuy Linh (Chemistry Institute, Vietnam Academy of Science and Technology, Vietnam) for providing S. aureus, Prof. Dr. Gen Larsson, Assc. Prof. Dr. Patrik Samuelson (The Division of Bioprocess Technology, KTH Biotechnology, Royal Institute of Technology, Stockholm, Sweden) and Dr.S.V.N.Vijayendra (Food Microbiology Dept., Central Food Technological Research Institute, Mysore-570020, India) for proofreading and correcting English in this manuscript. This study was carried out by facilities of National Key Laboratory of Gene Technology, Institute of Biotechnology, VAST, Vietnam.

\section{Authors' contribution}

NHT supervised the study and provided consultation. THD designed, analyzed data, wrote and proofread manuscript. TNL, TNN, NTT performed experiments. TNL wrote manuscript. SOE supervised on fermentation of recombinant strain.

\section{Funding/Support}

This study was supported by Ministry of Agriculture and Rural Development, Vietnam for the Project "Study on production of bio-antibacterial substances (nisin and enterocin) for food preservative" 20072009.

\section{References}

1. Calo-Mata P, Arlindo S, Boehme K, Miguel T, Pascoal A, Barros-Velazquez J. Current applications and future trends of lactic acid bacteria and their bacteriocins for the biopreservation of aquatic food products. Food Bioprocess Technol. 2007;1:43-63.doi:10.1007/s11947-007-0021-2

2. Drider D, Rebuffat S (Eds). Prokaryotic Antimicrobial Peptides. New York, NY: Springer New York; 2011.doi:10.1007/978-1-4419-7692-5

3. Cintas L, Casaus P, Havarstein L, Hernandez P, Nes I. Biochemical and genetic characterization of enterocin $\mathrm{P}$, a novel sec- dependent bacteriocin from Enterococcus faecium P13 with a broad antimicrobial spectrum. Appl Environ Microbiol. 1997;63:4321-4330.doi:10.1128/JB.182.23.68066814.2000

4. Herranz C, Driessen AJM. Sec-mediated secretion of bacteriocin Enterocin P by Lactococcus lactis. Appl Environ Microbiol. 2005;71:1959-1963.doi:10.1128/AEM.71.4.19591963.2005

5. Herranz C, Cintas LM, Hernandez PE, Moll GN, Driessen AJM: Enterocin $\mathrm{P}$ causes potassium ion efflux from Enterococcus faecium T136 cells. Antimicrob Agents Chemother. 2001; 45:901-904.doi:10.1128/AAC.45.3.901904.2001

6. Campos C, Rodriguez O, Calomata P, Prado M, Barrosvelazquez J. Preliminary characterization of bacteriocins from Lactococcus lactis, Enterococcus faecium and Enterococcus mundtii strains isolated from turbot (Psetta maxima). Food Res Int. 2006;39:356364.doi:10.1016/j.foodres.2005.08.008

7. Arlindo S, Calo P, Franco C, Prado M, Cepeda A, BarrosVelázquez J. Single nucleotide polymorphism analysis of the enterocin P structural gene of Enterococcus faecium strains isolated from nonfermented animal foods. Mol Nutr Food Res. 2006;50:1229-1238.doi:10.1002/mnfr.200600178

8. Gutiérrez J, Bourque D, Criado R, Choi YJ, Cintas LM, Hernández PE, Míguez CB. Heterologous extracellular production of enterocin P from Enterococcus faecium P13 in the methylotrophic bacterium Methylobacterium extorquens. FEMS Microbiol Lett. 2005;248:125-131.doi:http://dx.doi.org/10.1016/j.femsle.2005.05.029

9. Gutiérrez J, Criado R, Citti R, Martín M, Herranz C, Nes IF, Cintas LM, Hernández PE. Cloning, production and functional expression of enterocin $\mathrm{P}$, a sec-dependent bacteriocin produced by Enterococcus faecium P13, in Escherichia coli. Int J Food Microbiol. 2005;103:239-250.doi:10.1016/j.ijfoodmicro.2004.11.035

10. Cuozzo S, Calvez S, Prévost H, Drider D. Improvement of enterocin P purification process. Folia Microbiol (Praha). 
2006;51:401-405.doi:10.1007/BF02931583

11. Gutiérrez J, Criado R, Martin M, Herranz C, Cintas LM, Hernandez PE. Production of Enterocin P, an antilisterial pediocin-like bacteriocin from Enterococcus faecium P13, in Pichia pastoris. Antimicrob Agents Chemother. 2005;49:30043008.doi:10.1128/AAC.49.7.3004-3008.2005

12. Hackenberger CPR, Chen MM, Imperiali B. Expression of N-terminal Cys-protein fragments using an intein refolding strategy. Bioorg Med Chem. 2006;14:50435048.doi:10.1016/j.bmc.2006.03.003

13. Humphries HE, Christodoulides M, Heckels JE. Expression of the class 1 outer-membrane protein of Neisseria meningitidis in Escherichia coli and purification using a self-cleavable affinity tag. Protein Expr Purif. 2002;26:243-248.doi:10.1016/S10465928(02)00534-X

14. Evans TC Jr, Benner J, Xu MQ. The cyclization and polymerization of bacterially expressed proteins using modified self-splicing inteins. $J$ Biol Chem. 1999;274:1835918363.doi:10.1074/jbc.274.26.18359

15. Sydor JR, Mariano M, Sideris S, Nock S. Establishment of inteinmediated protein ligation under denaturing conditions: C-terminal labeling of a single-chain antibody for biochip screening. Bioconjug Chem. 2002;13:707-712.doi:10.1021/bc025534z

16. Chiang C-J, Chen H-C, Chao Y-P, Tzen JTC. Efficient system of artificial oil bodies for functional expression and purification of recombinant nattokinase in Escherichia coli. J Agric Food Chem. 2005;53:4799-4804.doi:10.1021/jf050264a

17. Barnard GC, McCool JD, Wood DW, Gerngross TU. Integrated recombinant protein expression and purification platform based on Ralstonia eutropha. Appl Environ Microbiol. 2005;71:5735-5742.doi:10.1128/AEM.71.10.57355742.2005

18. Schägger H, von Jagow G. Tricine-sodium dodecyl sulfatepolyacrylamide gel electrophoresis for the separation of proteins in the range from 1 to $100 \mathrm{kDa}$. Anallytical
Biochemistry.1987;166:368-379.doi:10.1016/00032697(87)90587-2

19. Bhunia AK, Johnson MG. A modified method to directly detect in SDS-PAGE the bacteriocin of Pediococcus acidilactici. Lett Appl Microbiol. 1992;15:5-7.doi:10.1111/j.1472-765X.1992.tb00709.x

20. Toba T, Yoshioka E, Itoh T. Lacticin, a bacteriocin produced by Lactobacillus delbrueckii subsp. lactis. Letters in Appl Microbiol. 1991;12:43-45.doi:10.1111/j.1472-765X.1991.tb00499.x

21. Jack RW, Tagg JR, Ray B. Bacteriocins of gram-positive bacteria. Microbiol Rev. 1995;59:171-200.

22. Sun Z, Chen J, Yao H, Liu L, Wang J, Zhang J, Liu J-N. Use of Ssp dnaB derived mini-intein as a fusion partner for production of recombinant human brain natriuretic peptide in Escherichia coli. Protein Expr Purif. 2005;43:2632.doi:10.1016/j.pep.2005.05.005

23. Nguyen TN, Le TN, Do TH, Tran NT, Pham TL, Le VT, Truong NH. TCloning and expression of enterocin $\mathrm{P}$ in the fusion form with CBD-SSP intein in Escherichia coli ER2566. J Biotecnol in Vietnam. 2009;7:27-33.

24. Pham TL, Le TN, Tran NT, Nguyen TN, Do TH, Le VT, Truong NH. Expression of gene coding for enterocin P of Enterococcus faecium in Pichia pastoris GS115. National Conference on Gennetic Modified Organisms and Biosecurity Management. 2009;145-149.

25. Nguyen TN, Pham TMD, Le TN, Le VT, Do TH, Tran NT, Pham TL, Truong NH. Construction of recombinant plasmid from expression vector $\mathrm{pAC} 7$ containing entP gene fused with fragments of BaAmyF1, BaAmyF2 for production of enterocin $\mathrm{P}$ in Bacillus subtilis 168. J Biotecnol in Vietnam. 2010;8:13-20.

26. Gutiérrez J, Larsen R, Cintas LM, Kok J, Hernández PE. Highlevel heterologous production and functional expression of the sec-dependent enterocin P from Enterococcus faecium P13 in Lactococcus lactis. Appl Microbiol Biotechnol. 2006;72:41-51.doi:10.1007/s00253-005-0233-1 